\title{
On the Assessment of Average Biosimilarity Based on a Three-Arm Parallel Design
}

\author{
Ginto Jacob Pottackal \\ Department of Mathematics and Statistics \\ University of Maryland Baltimore County \\ 1000 Hilltop Circle, Baltimore, Maryland 21250 \\ gintol@umbc.edu \\ Thomas Mathew \\ Department of Mathematics and Statistics \\ University of Maryland Baltimore County \\ 1000 Hilltop Circle, Baltimore, Maryland 21250 \\ mathew@umbc.edu
}

Received 23 November 2016

Accepted 30 May 2017

\begin{abstract}
Average biosimilarity is investigated under a three-arm parallel design: one arm corresponds to the test drug $T$, and the other two arms correspond to two versions of the reference drug, say $R_{1}$ and $R_{2}$. The hypothesis of interest is the equivalence of the population average response for $T$ with the mean of the population average responses for $R_{1}$ and $R_{2}$. The parameter of interest is formulated as the absolute difference of the above two averages, scaled by the absolute difference between the population means corresponding to $R_{1}$ and $R_{2}$. A difference parameter is also proposed. For the ratio parameter, a test can be derived using the asymptotic normality of an appropriate test statistic; however, the test is not satisfactory in terms of type I error probabilities. Improved tests are derived by applying a bootstrap calibration, and by using the idea of a generalized pivotal quantity (gpq). The tests are developed under equal variance and unequal variance scenarios. Sample size determination is also addressed. For the difference parameter, a satisfactory test is developed using the gpq idea. The proposed methods result in tests that are satisfactory in terms of type I error performance
\end{abstract}

Keywords: Bootstrap calibration; equivalence testing; generalized pivotal quantity; sample size determination

2000 Mathematics Subject Classification: 22E46, 53C35, 57S20

\section{Introduction}

Biosimilar drugs are highly similar products or imitations of already approved biological drugs. Unlike generic drugs, it is difficult to develop exact copies of biological products due to the complexity of the protein structure. For the approval of generic drug products, the commonly used method is to assess average bioequivalence $(\mathrm{ABE})$ regarding drug absorption through the conduct of bioequivalence studies. However, such a criterion alone may not be appropriate for concluding biosimilarity; nevertheless, the equivalence of averages should be a minimum requirement. We refer to the U.S. FDA guidance document [6] and the book by Chow [2] for further background information on biosimilars. In particular, Chow's book provides a thorough discussion, including a discussion of the various statistical criteria that can be used for establishing biosimilarity. 
The pharmacokinetic (PK) response that is typically used for assessing bioequivalence or biosimilarity consists of the area under the blood or plasma concentration time curve (AUC). Furthermore, for generating the data for the assessment of biosimilarity, parallel study designs are found to be more practical. Indeed, the FDA guidance document [6] recommends a parallel design for establishing biosimilarity. Since we are comparing two drugs, the original biotechnology drug (or reference drug, denoted by $R$ ), and a copy (the test drug, denoted by $T$ ), a two arm parallel design appears natural. Here the subjects are randomized between the two arms with one arm corresponding to the reference drug $R$, and the second arm corresponding to the test drug $T$. Since exact copies of biotechnology drugs are not possible, some authors suggest that $R$ should be compared with itself, and such information should be used to assess the similarity between $T$ and $R$; see the article by Kang and Chow [7]. These authors thus suggest a three-arm parallel design for assessing biosimilarity; one arm of the design corresponds to the test drug $T$, and other two arms correspond to the reference drug coming from two batches, or manufactured using two processes; we shall denote these two versions of the reference drug by $R_{1}$ and $R_{2}$. Subjects are randomized among the three arms. Three-arm trials for equivalence assessment are also discussed in Chang et al. [1]. However, the third arm considered by the authors is a placebo, unlike the three-arm trial considered in Kang and Chow [7].

The set up we shall use is the same as that in Kang and Chow [7]. Let $\mu_{T}, \mu_{R_{1}}$ and $\mu_{R_{2}}$ denote the population mean responses corresponding to $T, R_{1}$ and $R_{2}$. The responses could be $A U C$ or $C_{\max }$, very often after a log-transformation. In order to assess biosimilarity with respect to averages, the parameter suggested by Kang and Chow [7] is the ratio

$$
\theta=\left(\mu_{T}-\mu_{R}\right) /\left(\mu_{R_{1}}-\mu_{R_{2}}\right), \text { where } \mu_{R}=\left(\mu_{R_{1}}+\mu_{R_{2}}\right) / 2,
$$

and the hypotheses of interest are

$$
H_{0}:|\theta| \geq \delta, \text { vs } H_{1}:|\theta|<\delta
$$

for some specified threshold $\delta$. Biosimilarity is concluded with respect to averages if $H_{0}$ is rejected based on a statistical test. Here it is assumed that $\mu_{R_{1}} \neq \mu_{R_{2}}$, as in Kang and Chow [7]. The justification for choosing the parameter $\theta$ as defined above is that one cannot expect $\mu_{T}$ to be close to $\mu_{R}$ any more than the amount by which the population averages are close for two copies of $R$. However, there are other parameter choices that can capture this requirement; for example, we can consider the difference

$$
\theta_{1}=\left|\mu_{T}-\mu_{R}\right|-\left|\mu_{R_{1}}-\mu_{R_{2}}\right|
$$

where $\mu_{R}$ is as defined in (1.1). Now the hypotheses of interest are

$$
H_{0}:\left|\theta_{1}\right| \geq \delta_{1}, \text { vs } H_{1}:\left|\theta_{1}\right|<\delta_{1},
$$

for some specified threshold $\delta_{1}$. Biosimilarity is concluded with respect to averages if $H_{0}$ in (1.4) is rejected.

The purpose of our investigation is to develop appropriate tests for the hypothesis in (1.2) and (1.4). We shall assume normally distributed responses, as in Kang and Chow [7]. These authors have derived a test for the hypotheses in (1.2) using the asymptotic normality of the natural estimator of $\theta=\left(\mu_{T}-\mu_{R}\right) /\left(\mu_{R_{1}}-\mu_{R_{2}}\right)$, assuming a common variance for the responses from the test drug and the reference drug. Even under such a scenario, Kang and Chow's approximate test is not always 
satisfactory. We improve and generalize Kang and Chow's approach in the following ways. In the equal variance scenario, we apply a bootstrap calibration to the test due to Kang and Chow [7]. This results in a test that exhibits better performance in terms of type I error probability, but is still not entirely satisfactory. We also developed a test using the concept of a generalized pivotal quantity (gpq). The gpq-based test turned out to be the most satisfactory in terms of maintaining the type I error probability. We have also provided results on the power. We also consider the unequal variance situation, and pursue the same approaches to arrive at test procedures. We have also provided a table of sample sizes. The relevant results appear in Section 2 of the paper. Following this, we consider the hypotheses in (1.4) concerning the parameter $\theta_{1}=\left(\mu_{T}-\mu_{R}\right)-\left(\mu_{R_{1}}-\mu_{R_{2}}\right)$, and have investigated a gpq-based test. Simulation results show that such a test is satisfactory in terms of type I error probabilities. Details appear in Section 3 of the paper. Overall, our work has resulted in very satisfactory test procedures for assessing biosimilarity with respect to averages based on the hypotheses in (1.2) and (1.4). An example is presented in Section 4 of the paper. The example deals with testing the biosimilarity of Accofil, a biologic that is expected to be similar to the reference product Neupogen; the latter being used to boost white blood cell production in patients undergoing chemotherapy for certain cancers. Here we have a three arm design since Accofil will be compared to two versions of Neupogen: a version approved by the European Union and another version approved by the US. The relevant data are taken from a European Medicines Agency document [5]. Our methodology will be illustrated using this data set.

\section{Testing Average Biosimilarity Based on $\theta=\left(\mu_{T}-\mu_{R}\right) /\left(\mu_{R_{1}}-\mu_{R_{2}}\right)$}

Recall that our problem is that of testing the hypotheses in (1.2) based on data generated using a three-arm parallel design. Under such a design, let $n_{T}$ subjects receive the test drug $T, n_{R}$ subjects receive the reference drug $R_{1}$, and another group of $n_{R}$ subjects receive the reference drug $R_{2}$, where $R_{1}$ and $R_{2}$ represent two versions of the reference drug $R$ manufactured using two processes, or in two batches. In Kang and Chow [7], the authors assume a 2:1 ratio between $n_{T}$ and $n_{R}$. We shall also assume this, even though this is not essential for the development of our methodology. It is assumed that the responses follow a normal distribution where the population means corresponding to the three arms are denoted by $\mu_{T}, \mu_{R_{1}}$ and $\mu_{R_{2}}$, respectively, as specified in the previous section. In their work, Kang and Chow [7] assume a common population variance $\sigma^{2}$ for the responses from $T$, as well as for the responses from $R_{1}$ and $R_{2}$. In our investigation, we shall first consider the case of a common variance, and later relax this assumption. In other words, we shall also consider the case of a population variance $\sigma_{T}^{2}$ for the responses for $T$, and a population variance $\sigma_{R}^{2}$ for the responses from $R_{1}$, as well as for those from $R_{2}$, where $\sigma_{T}^{2}$ and $\sigma_{R}^{2}$ need not be equal. We shall also denote by $\bar{X}_{T}, \bar{X}_{R_{1}}$ and $\bar{X}_{R_{2}}$, respectively, the sample means based on the responses from the sample of size $n_{T}$ for $T$, the responses from the sample of size $n_{R}$ for $R_{1}$, and those from the sample of size $n_{R}$ for $R_{2}$. Furthermore, in the case of a common population variance $\sigma^{2}$, we shall denote by $S^{2}$ the unbiased estimator of $\sigma^{2}$ obtained by pooling the data from the three samples. We thus have the distributions

$$
\begin{aligned}
& \bar{X}_{T} \sim N\left(\mu_{T}, \frac{\sigma^{2}}{n_{T}}\right), \bar{X}_{R_{1}} \sim N\left(\mu_{R_{1}}, \frac{\sigma^{2}}{n_{R}}\right), \bar{X}_{R_{2}} \sim N\left(\mu_{R_{2}}, \frac{\sigma^{2}}{n_{R}}\right), \\
& \left(n_{T}+2 n_{R}-3\right) \frac{S^{2}}{\sigma^{2}} \sim \chi_{n_{T}+2 n_{R}-3}^{2},
\end{aligned}
$$


where $\chi_{m}^{2}$ denotes a central chisquare distribution with $m$ df. Furthermore, the above random variables are independent. In the case of unequal population variances, let $S_{T}^{2}$ denote the sample variance from the sample of $n_{T}$ responses for $T$, and let $S_{R}^{2}$ denote the sample variance obtained by pooling the $n_{R}$ responses for $R_{1}$ and the $n_{R}$ responses for $R_{2}$. We then have the distributions

$$
\begin{aligned}
& \bar{X}_{T} \sim N\left(\mu_{T}, \frac{\sigma_{T}^{2}}{n_{T}}\right), \bar{X}_{R_{1}} \sim N\left(\mu_{R_{1}}, \frac{\sigma_{R}^{2}}{n_{R}}\right), \bar{X}_{R_{2}} \sim N\left(\mu_{R_{2}}, \frac{\sigma_{R}^{2}}{n_{R}}\right), \\
& \left(n_{T}-1\right) \frac{S_{T}^{2}}{\sigma_{T}^{2}} \sim \chi_{n_{T}-1}^{2},\left(2 n_{R}-2\right) \frac{S_{R}^{2}}{\sigma_{R}^{2}} \sim \chi_{2 n_{R}-2}^{2},
\end{aligned}
$$

where the above random variables are also independent. In the common variance scenario, two test procedures are developed in Kang and Chow [7]: one based on the delta method, and a second test based on a linearization method. Based on numerical results, they then recommend the test based on the delta method, and also provide tables of sample sizes based on power considerations. In the common variance scenario, we shall first improve upon the delta method based test by applying a bootstrap calibration. Secondly, we shall extend the methodology to the unequal variance situation as well. In both cases, we shall also derive a test based on the idea of a generalized pivotal quantity. We shall also provide table of sample sizes so that our proposed tests will achieve a specified power.

\subsection{The generalized pivotal quantity (gpq)}

Before we describe the various test procedures, we shall briefly describe the generalized pivotal quantities that we shall use. The concept is due to Weerahandi [12]; see also [13] and [14]. A generalized pivotal quantity (gpq) is a function of the underlying random variables, and the observed data that are realizations of these random variables. We shall construct gpqs for $\mu_{T}, \mu_{R_{1}}$ and $\mu_{R_{2}}$, and then combine them to get a gpq for a parameter of interest (for example, the parameter $\theta$ in (1.1) and $\theta_{1}$ in (1.3)). A gpq is required to satisfy two properties: (i) given the observed data, its distribution is free of any unknown parameters, and (ii) when the random variables are replaced by the corresponding realizations (i.e., the observed data), the gpq simplifies to a quantity that is free of any nuisance parameters; very often, the simplified quantity is equal to the parameter of interest.

In the equal variance scenario (2.1), let $\bar{x}_{T}, \bar{x}_{R_{1}}, \bar{x}_{R_{2}}$ and $s^{2}$ denote the observed values of $\bar{X}_{T}, \bar{X}_{R_{1}}$, $\bar{X}_{R_{2}}$ and $S^{2}$, respectively. Then gpqs for $\mu_{T}, \mu_{R_{1}}$ and $\mu_{R_{2}}$, denoted by $\tilde{\mu}_{T}, \tilde{\mu}_{R_{1}}$ and $\tilde{\mu}_{R_{2}}$, respectively, are given by

$$
\begin{gathered}
\tilde{\mu}_{T}=\bar{x}_{T}-\frac{Z_{T}}{U / \sqrt{n_{T}+2 n_{R}-3}} \frac{s}{\sqrt{n_{T}}}, \\
\tilde{\mu}_{R_{1}}=\bar{x}_{R_{1}}+\frac{Z_{R_{1}}}{U / \sqrt{n_{T}+2 n_{R}-3}} \frac{s}{\sqrt{n_{R}}}, \\
\tilde{\mu}_{R_{2}}=\bar{x}_{R_{2}}+\frac{Z_{R_{2}}}{U / \sqrt{n_{T}+2 n_{R}-3}} \frac{s}{\sqrt{n_{R}}},
\end{gathered}
$$

where $Z_{T}, Z_{R_{1}}$ and $Z_{R_{2}}$ are independent standard normal random variables and $U^{2} \sim \chi^{2}$ with $\mathrm{df}=$ $n_{T}+2 n_{R}-3$. We refer to the book by Krishnamoorthy and Mathew [9], Section 1.4, for further details on the derivation of the above gpqs. Having obtained the gpqs in (2.3), a gpq for $\theta$, say $\tilde{\theta}$, 
can be obtained as

$$
\tilde{\theta}=\frac{\left|\tilde{\mu}_{T}-\tilde{\mu}_{R}\right|}{\left|\tilde{\mu}_{R_{1}}-\tilde{\mu}_{R_{2}}\right|}
$$

where $\tilde{\mu}_{R}=\left(\tilde{\mu}_{R_{1}}+\tilde{\mu}_{R_{2}}\right) / 2$. Percentiles of $\tilde{\theta}$ provide confidence limits for $\theta$, referred to as generalized confidence limits.

In the unequal variance scenario (2.2), gpqs for $\mu_{T}, \mu_{R_{1}}$ and $\mu_{R_{2}}$, denoted once again by $\tilde{\mu}_{T}, \tilde{\mu}_{R_{1}}$ and $\tilde{\mu}_{R_{2}}$, respectively, are given by

$$
\begin{gathered}
\tilde{\mu}_{T}=\bar{x}_{T}-\frac{Z_{T}}{U_{T} / \sqrt{n_{T}-1}} \frac{s_{T}}{\sqrt{n_{T}}}, \\
\tilde{\mu}_{R_{1}}=\bar{x}_{R_{1}}+\frac{Z_{R_{1}}}{U_{R} / \sqrt{2 n_{R}-2}} \frac{s_{R}}{\sqrt{n_{R}}}, \\
\tilde{\mu}_{R_{2}}=\bar{x}_{R_{2}}+\frac{Z_{R_{2}}}{U_{R} / \sqrt{2 n_{R}-2}} \frac{s_{R}}{\sqrt{n_{R}}},
\end{gathered}
$$

where $s_{T}^{2}$ and $s_{R}^{2}$ are the observed values of $S_{T}^{2}$ and $S_{R}^{2}$, respectively, $U_{T}^{2} \sim \chi^{2}$ with $\mathrm{df}=n_{T}-1$, $U_{R}^{2} \sim \chi^{2}$ with $\mathrm{df}=2 n_{R}-2$, and the rest of the quantities are as defined for the gpqs in (2.3).

\subsection{The case of a common variance}

We first give a brief description of the delta method approach given in Kang and Chow [7]. In view of the definition of $\theta$ in (1.1) and the distributions in (2.1), a natural estimator of $\theta$, say $\hat{\theta}$, is given by

$$
\hat{\theta}=\frac{\bar{X}_{T}-\left(\bar{X}_{R_{1}}+\bar{X}_{R_{2}}\right) / 2}{\bar{X}_{R_{1}}-\bar{X}_{R_{2}}} .
$$

Defining

$$
\mu_{1}=\mu_{T}-\left(\mu_{R_{1}}+\mu_{R_{2}}\right) / 2, \mu_{2}=\mu_{R_{1}}-\mu_{R_{2}}, \sigma_{1}^{2}=\frac{2 \sigma^{2}}{n_{T}}, \text { and } \sigma_{2}^{2}=\frac{2 \sigma^{2}}{n_{R}},
$$

$\hat{\theta}$ can also be expressed as

$$
\hat{\theta}=\frac{\bar{V}}{\bar{U}}, \text { where } \bar{V} \sim N\left(\mu_{1}, \sigma_{1}^{2}\right) \text { and } \bar{U} \sim N\left(\mu_{2}, \sigma_{2}^{2}\right),
$$

where we have used the assumption $n_{T}=2 n_{R}$. We also note that $\bar{V}$ and $\bar{U}$ are also independent. A straightforward application of the delta method gives

$$
\sqrt{n_{T}}\left(\frac{\bar{V}}{\bar{U}}-\frac{\mu_{1}}{\mu_{2}}\right) \sim N\left(0, \frac{2 \sigma^{2}}{\mu_{2}^{2}}+\frac{4 \mu_{1}^{2} \sigma^{2}}{\mu_{2}^{4}}\right),
$$

asymptotically. Following the methodology used in bioequivalence testing, we reject $H_{0}$ in (1.2) when

$$
Z=\frac{\left|\frac{\bar{V}}{\bar{U}}\right|-\delta}{\frac{S}{\sqrt{n_{T}}} \sqrt{\frac{2}{\bar{U}^{2}}+\frac{4 \bar{V}^{2}}{\bar{U}^{4}}}}<-z_{\alpha}
$$

where $S^{2}$ is the pooled variance mentioned in (2.1) and $z_{\alpha}$ is the upper $\alpha$ quantile of the standard normal distribution. Numerical results show that the performance of the above test is not satisfactory 
in terms of maintaining the type I error probability; the type I error probabilities are often higher than the nominal level. We shall thus employ a bootstrap calibration in order to improve the performance.

\subsubsection{Bootstrap Calibration}

The bootstrap calibration idea that we shall apply is taken from Chapter 18 in the book by Efron and Tibshirani [4]. We shall explain the calibration idea as it applies to our problem. Since we noted that the test procedure in (2.10) has type I error probability more than the nominal level, it is possible that the type I error probability can be made closer to $\alpha$ by carrying out the test using a significance level $\gamma<\alpha$. The required significance level $\gamma$ will be determined using the bootstrap. Under the normality assumption in (2.9), it is known that $\max _{H_{0}} P\left(Z<-z_{\alpha}\right)=P\left(Z<-z_{\alpha} \mid \theta=\delta\right)$, where $H_{0}$ is specified in (1.2). The above conclusion follows from the theoretical developments in the context of bioequivalence testing; see the book by Chow and Liu [3]. However, $P\left(Z<-z_{\alpha} \mid \theta=\delta\right)$ does depend on nuisance parameters. Since the type I error probability is being computed when $\theta=\mu_{1} / \mu_{2}=\delta$, the nuisance parameters in the model can be taken as $\lambda=\left(\mu_{1}, \sigma^{2}\right)$, so that $\mu_{2}$ is determined as $\mu_{2}=\mu_{1} / \delta$. Thus, when $\theta=\mu_{1} / \mu_{2}=\delta$, the distributions in (2.8) can be written as

$$
\bar{V} \sim N\left(\mu_{1}, \frac{2 \sigma^{2}}{n_{T}}\right) \text { and } \bar{U} \sim N\left(\mu_{1} / \delta, \frac{2 \sigma^{2}}{n_{R}}\right),
$$

where we have used the expressions for $\sigma_{1}^{2}$ and $\sigma_{2}^{2}$ given in (2.7). We note that based on (2.11), the estimator of $\mu_{1}$, say $\hat{\mu}_{10}$, is given by

$$
\hat{\mu}_{10}=\left(n_{T}+\frac{n_{R}}{\delta^{2}}\right)^{-1}\left(n_{T} \bar{V}+\frac{n_{R}}{\delta} \bar{U}\right) .
$$

We shall implement the bootstrap calibration by generating parametric bootstrap samples $\left(\bar{V}^{*}, \bar{U}^{*}, S^{*^{2}}\right)$ from the distributions

$$
\bar{V}^{*} \sim N\left(\hat{\mu}_{10}, \frac{2 S^{2}}{n_{T}}\right), \bar{U}^{*} \sim N\left(\hat{\mu}_{10} / \delta, \frac{2 S^{2}}{n_{R}}\right), \text { and }\left(n_{T}+2 n_{R}-3\right) \frac{S^{*^{2}}}{S^{2}} \sim \chi_{n_{T}+2 n_{R}-3}^{2},
$$

where $S^{2}$ is as specified in (2.1), and $\hat{\mu}_{10}$ is given in (2.12). Now let $\left(\bar{V}_{i}^{*}, \bar{U}_{i}^{*}, S_{i}^{*^{2}}\right), i=1,2, \ldots, B$, be a parametric bootstrap sample of size $B$ generated from the distribution of $\left(\bar{V}^{*}, \bar{U}^{*}, S^{*^{2}}\right)$ given in (2.13). Let

$$
Z_{i}^{*}=\frac{\left|\frac{\bar{V}_{i}^{*}}{\bar{U}_{i}^{*}}\right|-\delta}{\frac{S_{i}^{*}}{\sqrt{n_{T}}} \sqrt{\frac{2}{\bar{U}_{i}^{* 2}}+\frac{4 \overline{4}_{i}^{*}}{\bar{U}_{i}^{* 4}}}}
$$

$i=1,2, \ldots, B$. Our objective is to choose a $\gamma$ that will make the type I error probability very close to $\alpha$. For this, we can consider a grid of values of $\gamma$, and compute the proportion of times $Z_{i}^{*}<-z_{\gamma}$. Now pick a value of $\gamma$ for which this proportion is equal to $\alpha$. We used the $R$ function uniroot to obtain $\gamma$. Let the resulting choice of $\gamma$ be denoted by $\hat{\alpha}$ in order to emphasize that the significance level to be used is actually estimated from the data. The bootstrap calibrated test consists of rejecting $H_{0}$ in (1.2) when $Z<-z_{\hat{\alpha}}$, where $Z$ is defined in (2.10). 


\subsubsection{The gpq-based test}

As noted in Section 2.1, a gpq for $\theta$ is the quantity $\tilde{\theta}$ given in (2.4). In order to test (1.2) at significance level $\alpha$, we compute the $100(1-\alpha)$ th percentile of $\tilde{\theta}$, and reject $H_{0}$ if such a percentile is less than $\delta$. Note that in order to compute such a percentile, we keep the observed data $\left(\bar{x}_{T}, \bar{x}_{R_{1}}, \bar{x}_{R_{2}}\right.$ and $s^{2}$ ) as fixed, and treat the gpq as a random variable that is a function of the independent standard normal random variables $Z_{T}, Z_{R_{1}}$ and $Z_{R_{2}}$, and the chisquare random variable $U^{2} \sim \chi^{2}$ with $\mathrm{df}=$ $n_{T}+2 n_{R}-3$. Several copies of the gpq can be generated by generating several values of $\left(Z_{T}, Z_{R_{1}}\right.$, $\left.Z_{R_{2}}, U^{2}\right)$. The $100(1-\alpha)$ th percentile of the gpq can be computed based on such generated values, which can then be used to carry out the test.

\subsubsection{Numerical Results}

In order to assess the performance of the delta method based test proposed by Kang and Chow [7] along with the improvement resulting from bootstrap calibration, and to compared with the gpq based test, we shall now report some simulation results. The sample sizes and parameter combinations used in the simulations are taken from Kang and Chow [7]. We used the sample sizes $n_{T}=30$, 50, and 100, and $n_{R}=n_{T} / 2$. Two values of $\delta$ were considered: $\delta=1.1$ and 1.2, and three values of $\sigma$ were considered: $\sigma=1,2$, and 3. Table 1 gives the type I error probabilities of the test with rejection region (2.10), its bootstrap calibrated version, and the gpq based test. In the table, these are denoted by "Delta method", "Bootstrap calibration" and "GPQ Method", respectively. We have used a 5\% nominal level, and the results in Table 1 are based on 10,000 simulations.

From the numerical results, it should be clear that the delta method based test exhibits poor performance when the sample size is small and/or when the common variance $\sigma^{2}$ is large. The bootstrap calibration improves the type I error performance of the test in terms of providing type I error probabilities close to the nominal level. However, the test is still not satisfactory. On the other hand, the gpq based test appears to provide satisfactory type I error probabilities in all scenarios considered for simulation. Clearly, the test to be recommended is the gpq based test.

Table 1 . Type 1 error probabilities of the different tests in the equal variance case for a $5 \%$ significance level.

\begin{tabular}{|c|c|c|c|c|c|c|c|c|c|c|c|c|c|c|c|c|}
\hline$\delta$ & $\mu_{T}$ & $\mu_{R 1}$ & $\mu_{R 2}$ & $\sigma^{2}$ & $n_{T}$ & $\begin{array}{l}\text { Delta } \\
\text { Method }\end{array}$ & $\begin{array}{l}\text { Bootstrap } \\
\text { Calibration }\end{array}$ & $\begin{array}{l}\text { GPQ } \\
\text { Method }\end{array}$ & $\mu_{T}$ & $\mu_{R 1}$ & $\mu_{R 2}$ & $\sigma^{2}$ & $n_{T}$ & $\begin{array}{l}\text { Delta } \\
\text { Method }\end{array}$ & $\begin{array}{l}\text { Bootstrap } \\
\text { Calibration }\end{array}$ & $\begin{array}{l}\text { GPQ } \\
\text { Method }\end{array}$ \\
\hline \multirow{3}{*}{1.2} & \multirow{3}{*}{117} & \multirow{3}{*}{100} & \multirow{3}{*}{110} & \multirow{3}{*}{1} & 30 & 0.0631 & 0.0447 & 0.0441 & \multirow{3}{*}{110.2} & \multirow{3}{*}{106} & \multirow{3}{*}{100} & \multirow{3}{*}{1} & 30 & 0.0686 & 0.0535 & 0.0478 \\
\hline & & & & & 50 & 0.0564 & 0.0434 & 0.0524 & & & & & 50 & 0.0621 & 0.0499 & 0.0466 \\
\hline & & & & & 100 & 0.0578 & 0.0477 & 0.0523 & & & & & 100 & 0.0581 & 0.0485 & 0.0543 \\
\hline \multirow{3}{*}{1.2} & \multirow{3}{*}{117} & \multirow{3}{*}{100} & \multirow{3}{*}{110} & \multirow{3}{*}{2} & 30 & 0.0647 & 0.0397 & 0.0476 & \multirow{3}{*}{110.2} & \multirow{3}{*}{106} & \multirow{3}{*}{100} & \multirow{3}{*}{2} & 30 & 0.0726 & 0.0519 & 0.0456 \\
\hline & & & & & 50 & 0.0557 & 0.0366 & 0.0499 & & & & & 50 & 0.0701 & 0.0523 & 0.0501 \\
\hline & & & & & 100 & 0.0562 & 0.0441 & 0.0491 & & & & & 100 & 0.0583 & 0.0477 & 0.0511 \\
\hline \multirow{3}{*}{1.2} & \multirow{3}{*}{117} & \multirow{3}{*}{100} & \multirow{3}{*}{110} & \multirow{3}{*}{3} & 30 & 0.0694 & 0.0396 & 0.0453 & \multirow{3}{*}{110.2} & \multirow{3}{*}{106} & \multirow{3}{*}{100} & \multirow{3}{*}{3} & 30 & 0.0754 & 0.0492 & 0.0488 \\
\hline & & & & & 50 & 0.0606 & 0.0363 & 0.0451 & & & & & 50 & 0.0696 & 0.0509 & 0.0451 \\
\hline & & & & & 100 & 0.0563 & 0.0404 & 0.0515 & & & & & 100 & 0.0630 & 0.0502 & 0.0479 \\
\hline \multirow{3}{*}{1.1} & \multirow{3}{*}{116} & \multirow{3}{*}{100} & \multirow{3}{*}{110} & & 30 & 0.0590 & 0.0370 & 0.0461 & & & & & 30 & 0.0656 & 0.0490 & 0.0460 \\
\hline & & & & 1 & 50 & 0.0592 & 0.0435 & 0.0498 & 109.6 & 106 & 100 & 1 & 50 & 0.0640 & 0.0527 & 0.0468 \\
\hline & & & & & 100 & 0.0553 & 0.0455 & 0.0512 & & & & & 100 & 0.0605 & 0.0517 & 0.0470 \\
\hline & & & & & 30 & 0.0637 & 0.0379 & 0.0457 & & & & & 30 & 0.0695 & 0.0478 & 0.0472 \\
\hline 1.1 & 116 & 100 & 110 & 2 & 50 & 0.0614 & 0.0407 & 0.0492 & 109.6 & 106 & 100 & 2 & 50 & 0.0671 & 0.0531 & 0.0510 \\
\hline & & & & & 100 & 0.0606 & 0.0435 & 0.0481 & & & & & 100 & 0.0615 & 0.0510 & 0.0457 \\
\hline & & & & & 30 & 0.0646 & 0.0498 & 0.0468 & & & & & 30 & 0.0767 & 0.0510 & 0.0459 \\
\hline 1.1 & 116 & 100 & 110 & 3 & 50 & 0.0659 & 0.0528 & 0.0476 & 109.6 & 106 & 100 & 3 & 50 & 0.0688 & 0.0497 & 0.0515 \\
\hline & & & & & 100 & 0.0610 & 0.0530 & 0.0470 & & & & & 100 & 0.0644 & 0.0503 & 0.0497 \\
\hline
\end{tabular}



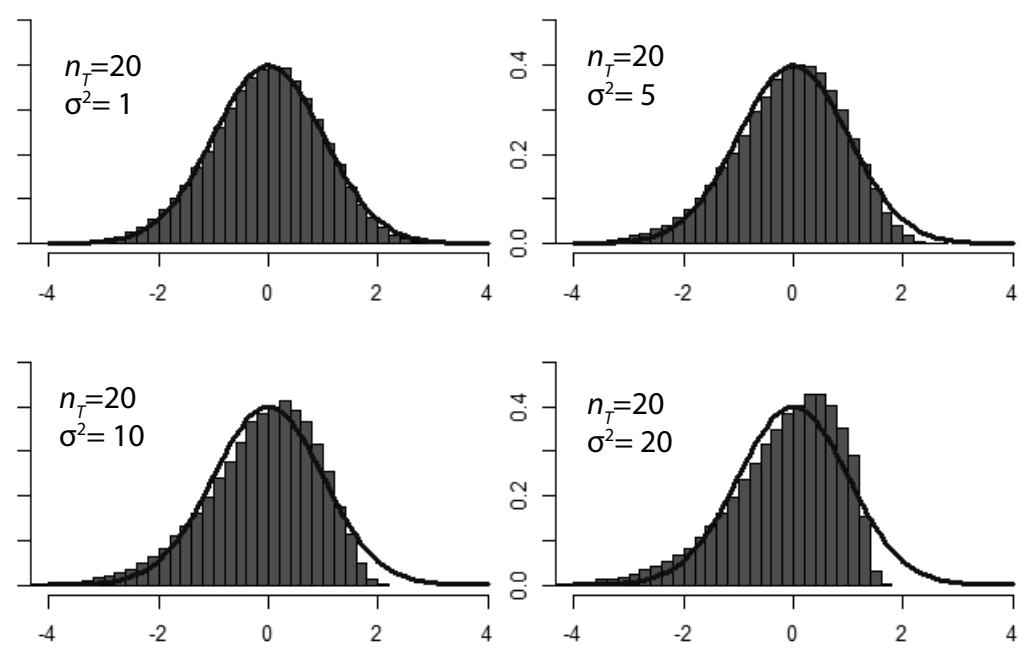

Fig. 1. Distribution of the test statistic under different variances and $n_{R}=20$

Table 2. Upper and Lower tail probabilities of the test statistic $Z$ in (2.10) (based on 100,000

\begin{tabular}{|c|c|c|c|c|c|c|c|c|}
\hline \multirow{4}{*}{\multicolumn{2}{|c|}{$\sigma$}} & & \multirow{2}{*}{\multicolumn{3}{|c|}{$\alpha=0.05$}} & & & \\
\hline & & \multirow{3}{*}{ Probability } & & & & \multicolumn{3}{|c|}{$\alpha=0.1$} \\
\hline & & & \multicolumn{3}{|c|}{$n_{R}$} & \multicolumn{3}{|c|}{$n_{R}$} \\
\hline & & & 20 & 50 & 100 & 20 & 50 & 100 \\
\hline \multirow{6}{*}{ simulations) } & \multirow{2}{*}{1} & $\mathrm{P}\left(Z<-z_{\alpha}\right)$ & 0.0592 & 0.0560 & 0.0545 & 0.1108 & 0.1085 & 0.1029 \\
\hline & & $\mathrm{P}\left(Z>z_{\alpha}\right)$ & 0.0382 & 0.0426 & 0.0453 & 0.0876 & 0.0911 & 0.0938 \\
\hline & \multirow{2}{*}{5} & $\mathrm{P}\left(Z<-z_{\alpha}\right)$ & 0.0721 & 0.0641 & 0.0596 & 0.1243 & 0.1143 & 0.1103 \\
\hline & & $\mathrm{P}\left(Z>z_{\alpha}\right)$ & 0.0227 & 0.0333 & 0.0379 & 0.0695 & 0.0813 & 0.0887 \\
\hline & \multirow{2}{*}{10} & $\mathrm{P}\left(Z<-z_{\alpha}\right)$ & 0.0785 & 0.0685 & 0.0641 & 0.1292 & 0.1190 & 0.1140 \\
\hline & & $\mathrm{P}\left(Z>z_{\alpha}\right)$ & 0.0095 & 0.0256 & 0.0331 & 0.0524 & 0.0743 & 0.0842 \\
\hline
\end{tabular}

In order to have further insight into the poor performance of the delta method based test, we plotted a histogram of the test statistic based on 10,000 simulated values for $n_{R}=20$, and $\sigma^{2}=1,5$, 10 and 20. These appear in Figure 1, with the normal curve superimposed. It is clear that the normal approximation is poor as $\sigma$ becomes large. In Table 2, we have given the tail probabilities of the distribution of the test statistic, below and above $-z_{\alpha}$ and $z_{\alpha}$, where $z_{\alpha}$ is the upper $\alpha$ percentile of the standard normal distribution. The asymmetry of the distribution and the poor quality of the normal approximation should be clear from Table 2 when the sample size is small and/or $\sigma^{2}$ is large.

\subsubsection{Power comparison}

Figure 2 gives plots of the power curves of the three tests, plotted against the sample size, for $\sigma^{2}$ $=1$. For this, the null value is chosen as $\theta=\delta=1.2$ and the alternative used is $\theta=0$. For each combination of the sample size and $\sigma$, the power was obtained using $5000 \times 5000$ simulations. We note that for small sample sizes, the delta method based test has a slightly larger power, and the gpq 


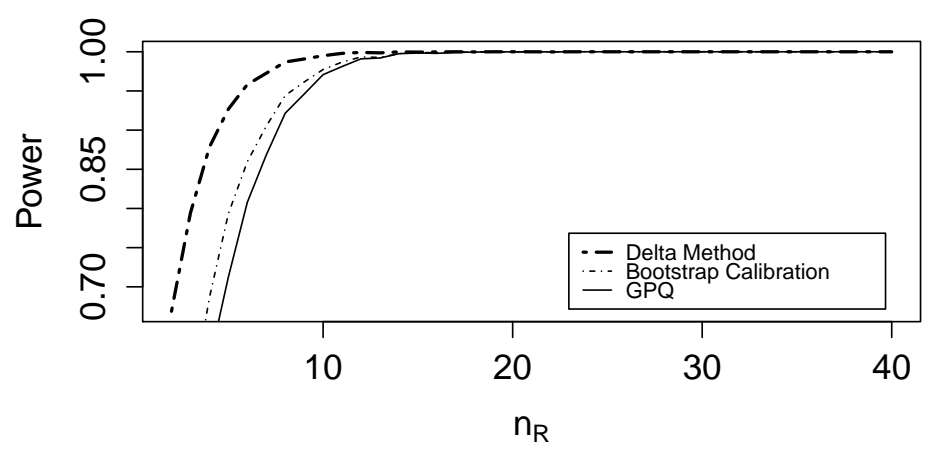

Fig. 2. Power functions plotted against the sample size for the alternative $\theta=0$ when $\sigma_{T}^{2}=\sigma_{R}^{2}=\sigma^{2}=1$.

based test has somewhat lower power. This is to be expected since the type I error probabilities are slightly inflated for the delta method based test (as noted in Table 1). However, as the sample size gets large, the difference in power among the three tests disappears. More extensive plots are given in Pottackal [10], and the pattern noted in the different plots is the same as that in Figure 2.

\subsection{The case of unequal variances}

We now have the distributions specified in (2.2). A natural estimator of $\theta$ is once again given by $\hat{\theta}$ in (2.6). Defining

$$
\mu_{1}=\mu_{T}-\left(\mu_{R_{1}}+\mu_{R_{2}}\right) / 2, \mu_{2}=\mu_{R_{1}}-\mu_{R_{2}}, \sigma_{1}^{2}=\frac{\sigma_{T}^{2}+\sigma_{R}^{2}}{n_{T}} \text { and } \sigma_{2}^{2}=\frac{2 \sigma_{R}^{2}}{n_{R}},
$$

$\hat{\theta}$ can also be expressed as

$$
\hat{\theta}=\frac{\bar{V}}{\bar{U}}, \text { where } \bar{V} \sim N\left(\mu_{1}, \sigma_{1}^{2}\right) \text { and } \bar{U} \sim N\left(\mu_{2}, \sigma_{2}^{2}\right),
$$

where we have used the assumption $n_{T}=2 n_{R}$. Note that $\bar{V}$ and $\bar{U}$ are also independent. Once again, a straightforward application of the delta method gives

$$
\sqrt{n_{T}}\left(\frac{\bar{V}}{\bar{U}}-\frac{\mu_{1}}{\mu_{2}}\right) \sim N\left(0, \frac{\sigma_{1}^{2}}{\mu_{2}^{2}}+\frac{\mu_{1}^{2} \sigma_{2}^{2}}{\mu_{2}^{4}}\right)
$$

asymptotically. We reject $H_{0}$ when

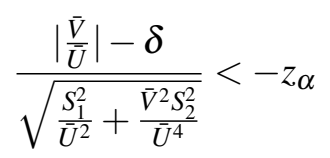

where $S_{1}^{2}$ and $S_{2}^{2}$ are the variances given in (2.2). A bootstrap calibrated version can also be derived as done earlier for the case of equal variances. For this, we note that with $\sigma_{1}^{2}$ and $\sigma_{2}^{2}$ as defined in (2.15), respective estimates $\hat{\sigma}_{1}^{2}$ and $\hat{\sigma}_{2}^{2}$ are given by $\hat{\sigma}_{1}^{2}=\left(S_{T}^{2}+S_{R}^{2}\right) / n_{T}$ and $\hat{\sigma}_{2}^{2}=2 S_{R}^{2} / n_{R}$, where 
$S_{T}^{2}$ and $S_{R}^{2}$ have the distributions specified in (2.2). In view of (2.16), a derivation similar to (2.12) gives us

$$
\hat{\mu}_{10}=\left(\frac{1}{\hat{\sigma}_{1}^{2}}+\frac{1}{\delta^{2} \hat{\sigma}_{2}^{2}}\right)^{-1}\left(\frac{\bar{V}}{\hat{\sigma}_{1}^{2}}+\frac{\bar{U}}{\delta \hat{\sigma}_{2}^{2}}\right) .
$$

In order to perform the bootstrap calibration, parametric bootstrap samples are generated from the distributions

$$
\bar{V}^{*} \sim N\left(\hat{\mu}_{10}, \hat{\sigma}_{1}^{2}\right), \bar{U}^{*} \sim N\left(\hat{\mu}_{10} / \delta, \hat{\sigma}_{2}^{2}\right),\left(n_{T}-1\right) \frac{S_{T}^{* 2}}{S_{T}^{2}} \sim \chi_{n_{T}-1}^{2},\left(2 n_{R}-2\right) \frac{S_{R}^{* 2}}{S_{R}^{2}} \sim \chi_{2 n_{R}-2}^{2} .
$$

Furthermore, a gpq based test can also be derived. We recall that the derivation of the gpqs in the unequal variance scenario is explained in Section 2.1.

\subsubsection{Type I error and power}

Similar to Table 1 and Figure 2, Table 3 and Figure 3, respectively, give the type I error probabilities and power plots for the different tests in the unequal variance scenario for the sample sizes $n_{T}=$ 30,50 , and 100, $n_{R}=n_{T} / 2$, and null value $\delta=1.2$. Furthermore, the $\sigma_{T}^{2}$ and $\sigma_{R}^{2}$ were chosen to take the values 1, 2 and 3 . The power plots have been obtained at the alternative value $\theta=0$, and is given only for the parameter choice $\sigma_{T}^{2}=1$ and $\sigma_{R}^{2}=2$ (see Pottackal [10] for further plots on the

Table 3. Type 1 error probabilities of the different tests in the unequal variance case for a 5\%

\begin{tabular}{|c|c|c|c|c|c|c|c|c|c|}
\hline$\delta$ & $\mu_{T}$ & $\mu_{R 1}$ & $\mu_{R 2}$ & $\sigma_{T}^{2}$ & $\sigma_{R}^{2}$ & $n_{T}$ & $\begin{array}{c}\text { Delta } \\
\text { Method }\end{array}$ & $\begin{array}{c}\text { Bootstrap } \\
\text { Calibration }\end{array}$ & $\begin{array}{c}\text { GPQ } \\
\text { Method }\end{array}$ \\
\hline \multirow{3}{*}{1.2} & \multirow{3}{*}{117} & \multirow{3}{*}{100} & \multirow{3}{*}{110} & \multirow{3}{*}{1} & \multirow{3}{*}{1} & 30 & 0.0629 & 0.0380 & 0.0479 \\
\hline & & & & & & 50 & 0.0595 & 0.0409 & 0.0484 \\
\hline & & & & & & 100 & 0.0550 & 0.0429 & 0.0516 \\
\hline \multirow{3}{*}{1.2} & \multirow{3}{*}{117} & \multirow{3}{*}{100} & \multirow{3}{*}{110} & \multirow{3}{*}{1} & \multirow{3}{*}{2} & 30 & 0.0687 & 0.0405 & 0.0509 \\
\hline & & & & & & 50 & 0.0634 & 0.0418 & 0.0468 \\
\hline & & & & & & 100 & 0.0579 & 0.0430 & 0.0498 \\
\hline \multirow{3}{*}{1.2} & \multirow{3}{*}{117} & \multirow{3}{*}{100} & \multirow{3}{*}{110} & \multirow{3}{*}{1} & \multirow{3}{*}{3} & 30 & 0.0694 & 0.0393 & 0.0476 \\
\hline & & & & & & 50 & 0.0641 & 0.0412 & 0.0510 \\
\hline & & & & & & 100 & 0.0620 & 0.0442 & 0.0532 \\
\hline \multirow{3}{*}{1.2} & \multirow{3}{*}{117} & \multirow{3}{*}{100} & \multirow{3}{*}{110} & \multirow{3}{*}{2} & \multirow{3}{*}{1} & 30 & 0.0618 & 0.0289 & 0.0463 \\
\hline & & & & & & 50 & 0.0579 & 0.0320 & 0.0491 \\
\hline & & & & & & 100 & 0.0584 & 0.0381 & 0.0497 \\
\hline \multirow{3}{*}{1.2} & \multirow{3}{*}{117} & \multirow{3}{*}{100} & \multirow{3}{*}{110} & \multirow{3}{*}{2} & \multirow{3}{*}{2} & 30 & 0.0637 & 0.0332 & 0.0500 \\
\hline & & & & & & 50 & 0.0603 & 0.0353 & 0.0526 \\
\hline & & & & & & 100 & 0.0563 & 0.0391 & 0.0511 \\
\hline & & & & & & 30 & 0.0682 & 0.0514 & 0.0491 \\
\hline 1.2 & 117 & 100 & 110 & 2 & 3 & 50 & 0.0622 & 0.0523 & 0.0508 \\
\hline & & & & & & 100 & 0.0604 & 0.0506 & 0.0474 \\
\hline & & & & & & 30 & 0.0619 & 0.0301 & 0.0526 \\
\hline 1.2 & 117 & 100 & 110 & 3 & 1 & 50 & 0.0561 & 0.0266 & 0.0501 \\
\hline & & & & & & 100 & 0.0546 & 0.0266 & 0.0475 \\
\hline & & & & & & 30 & 0.0657 & 0.0285 & 0.0487 \\
\hline 1.2 & 117 & 100 & 110 & 3 & 2 & 50 & 0.0610 & 0.0317 & 0.0500 \\
\hline & & & & & & 100 & 0.0572 & 0.0363 & 0.0479 \\
\hline & & & & & & 30 & 0.0667 & 0.0302 & 0.0499 \\
\hline 1.2 & 117 & 100 & 110 & 3 & 3 & 50 & 0.0635 & 0.0352 & 0.0512 \\
\hline & & & & & & 100 & 0.0586 & 0.0391 & 0.0519 \\
\hline
\end{tabular}
significance level. 


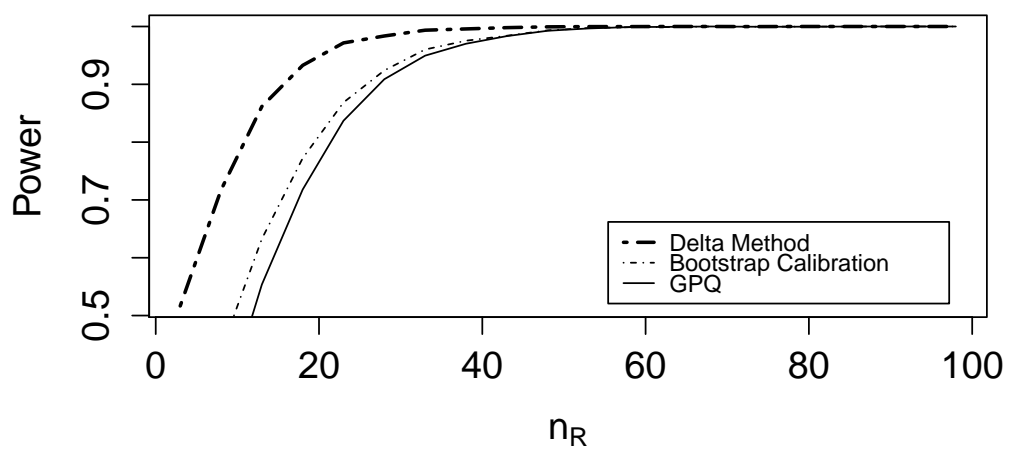

Fig. 3. Power functions plotted against the sample size for the alternative $\theta=0$ when $\sigma_{T}^{2}$ and $\sigma_{R}^{2}$ are unequal, with $\sigma_{T}^{2}$ $=1$ and $\sigma_{R}^{2}=2$.

power). The conclusions from Table 3 and Figure 3 are similar to those for the equal variance case. In particular, the gpq based test emerges as the one that is most satisfactory.

\subsubsection{Sample size calculation}

In their paper, Kang and Chow [7] have provided table of sample sizes so that the delta method based test will provide $80 \%$ and $90 \%$ power. Table 4 gives the sample sizes $n_{T}$ that will guarantee $90 \%$ power by the different tests in the unequal variance scenario.

Note that once $n_{T}$ is determined, $n_{R}$ is obtained as $2 n_{T}$. From Table 4 , we see that overall, the required sample size is slightly higher for the gpq based test. This is to be expected in view of the type I error performance of the tests.

Here we would like to point out that sample size determination in the context of equivalence trials is discussed in several articles; see [1] and [11]. Also, Kang and Kim [8] have addressed the sample size issue for biosimilar products; however, the set up considered by the authors is the usual bioequivalence scenario involving a single test drug and a single reference drug.

Table 4. Sample sizes $n_{T}$ necessary to guarantee $90 \%$ power for the delta method based test, its bootstrap calibrated version and the gpq based test at the alternative value $\theta=0$, for a $5 \%$ significance level.

\begin{tabular}{|c|c|c|c|c|}
\hline$\sigma_{T}^{2}$ & $\sigma_{R}^{2}$ & $\begin{array}{c}\text { Delta } \\
\text { Method }\end{array}$ & $\begin{array}{c}\text { Bootstrap } \\
\text { Calibration }\end{array}$ & $\begin{array}{c}\text { GPQ } \\
\text { Method }\end{array}$ \\
\hline 1 & 1 & 5 & 8 & 8 \\
2 & 2 & 9 & 12 & 13 \\
3 & 3 & 12 & 18 & 21 \\
1 & 2 & 19 & 27 & 29 \\
1 & 3 & 23 & 37 & 39 \\
1 & 4 & 29 & 49 & 51 \\
\hline
\end{tabular}




\section{Testing Average Biosimilarity Based on $\left|\mu_{T}-\mu_{R}\right|-\left|\mu_{R_{1}}-\mu_{R_{2}}\right|$}

We note that the hypothesis in (1.2), formulated in terms of the parameter $\theta$ defined in (1.1), is what is given in Kang and Chow [7]. We have simply followed their formulation, and have improved their test using bootstrap calibration, suggested a test based on the gpq, and have also extended their results to the unequal variance scenario. It should however be noted that if the means $\mu_{R_{1}}$ and $\mu_{R_{2}}$ are very close, $\theta$ defined in (1.1) will have its denominator close to zero. This could present practical difficulties; for example, how can one choose the threshold $\delta$ in the hypotheses in (1.2), when $\mu_{R_{1}}$ and $\mu_{R_{2}}$ are unknown and their difference could be close to zero? It appears that an alternative formulation could mitigate this problem. Thus, instead of the ratio used to define $\theta$ in (1.1), we shall consider the parameter to be the difference defined in (1.3) and consider the hypotheses

$$
H_{0}: \theta_{1} \geq \delta_{1}, \text { vs } H_{1}: \theta_{1}<\delta_{1}
$$

for some specified threshold $\delta_{1}$. Note that $\theta_{1}$ also measures the magnitude of $\left|\mu_{T}-\mu_{R}\right|$ relative to that of $\left|\mu_{R_{1}}-\mu_{R_{2}}\right|$. We shall develop a test for the hypotheses in (3.1) using the gpq approach. Let $\tilde{\mu}_{T}, \tilde{\mu}_{R_{1}}$ and $\tilde{\mu}_{R_{2}}$, respectively denote gpqs for $\mu_{T}, \mu_{R_{1}}$ and $\mu_{R_{2}}$, as defined in Section 2.1. We recall that the definition of these quantities depend on whether the variances $\sigma_{T}^{2}$ and $\sigma_{R}^{2}$ are equal or unequal. Also define $\tilde{\mu}_{R}=\left(\tilde{\mu}_{R_{1}}+\tilde{\mu}_{R_{2}}\right) / 2$. A gpq for $\theta_{1}$ defined in (1.3) is the quantity $\tilde{\theta}_{1}$ given by

$$
\tilde{\theta}_{1}=\left|\tilde{\mu}_{T}-\tilde{\mu}_{R}\right|-\left|\tilde{\mu}_{R_{1}}-\tilde{\mu}_{R_{2}}\right|
$$

and the hypotheses in (3.1) can be tested using the 100(1- $\alpha)$ th percentile of the resulting gpq. An estimate of this percentile can be easily obtained using Monte Carlo simulation, and we reject $H_{0}$ if the $100(1-\alpha)$ th percentile so obtained is less than $\delta_{1}$. Table 5 and Table 6 give the type I error probabilities of the resulting test in the equal variance and unequal variance scenarios. It should be clear that the gpq based test is quite accurate.

Table 5. Type 1 error probabilities of the gpq based test for testing the hypotheses in (3.1) at a 5\% significance level $\left(\sigma_{T}^{2}=\sigma_{R}^{2}\right)$.

\begin{tabular}{|llll|lll|}
\hline \multirow{2}{*}{$\mu_{T}$} & \multirow{2}{*}{$\mu_{R 1}$} & \multirow{2}{*}{$\mu_{R 2}$} & $\sigma_{T}^{2}$ & \multicolumn{3}{|c|}{$n$} \\
\cline { 5 - 7 } 117 & \multirow{2}{*}{100} & \multirow{2}{*}{110} & 2 & 0.0465 & 0.0502 & 0.0500 \\
& & & 3 & 0.0499 & 0.0486 & 0.0463 \\
\hline \multirow{3}{*}{116} & \multirow{2}{*}{100} & \multirow{2}{*}{110} & 2 & 0.0477 & 0.0479 & 0.0499 \\
& & & 3 & 0.0500 & 0.0479 & 0.0493 \\
\hline \multirow{3}{*}{110.2} & \multirow{2}{*}{106} & \multirow{2}{*}{100} & 2 & 0.0465 & 0.0464 & 0.0522 \\
& & & 3 & 0.0499 & 0.0477 & 0.0526 \\
\hline \multirow{3}{*}{109.6} & \multirow{2}{*}{106} & \multirow{2}{*}{100} & 2 & 0.0469 & 0.0491 & 0.0490 \\
& & & 3 & 0.0491 & 0.0482 & 0.0507 \\
\hline
\end{tabular}


Table 6. Type 1 error probabilities of the gpq based test for testing the hypotheses in (3.1) at a 5\% significance level $\left(\sigma_{T}^{2}\right.$ and $\sigma_{R}^{2}$ are unequal and $\left.\sigma_{T}^{2}=1\right)$.

\begin{tabular}{|llll|lll|}
\hline \multirow{2}{*}{$\mu_{T}$} & \multirow{2}{*}{$\mu_{R 1}$} & \multirow{2}{*}{$\mu_{R 2}$} & \multirow{2}{*}{$\sigma_{R}^{2}$} & \multicolumn{3}{|c|}{$n$} \\
\cline { 5 - 7 } & & & & 30 & 50 & \\
\hline \multirow{3}{*}{117} & \multirow{2}{*}{100} & \multirow{2}{*}{110} & 2 & 0.0459 & 0.0510 & 0.0475 \\
& & & 3 & 0.0524 & 0.0502 & 0.0461 \\
\hline \multirow{2}{*}{116} & \multirow{2}{*}{100} & \multirow{2}{*}{110} & 2 & 0.0486 & 0.0496 & 0.0501 \\
& & & 3 & 0.0479 & 0.0483 & 0.0483 \\
\hline \multirow{3}{*}{110.2} & \multirow{2}{*}{106} & \multirow{2}{*}{100} & 2 & 0.0484 & 0.0510 & 0.0504 \\
& & & 3 & 0.0495 & 0.0506 & 0.0502 \\
\hline \multirow{3}{*}{109.6} & \multirow{2}{*}{106} & \multirow{2}{*}{100} & 2 & 0.0478 & 0.0491 & 0.0503 \\
& & & 3 & 0.0486 & 0.0527 & 0.0468 \\
\hline
\end{tabular}

\section{An Example}

The example is taken from the European Medicines Agency document [5]. The problem addressed in the document is that of testing biosimilarity of Accofil, a biologic that is expected to be similar to the reference product Neupogen. The biotechnology drug Neupogen has been approved for use to boost white blood cell production in patients undergoing chemotherapy for certain cancers. Two versions, say $R_{1}$ and $R_{2}$, of Neupogen are used in the biosimilarity study: EU-approved Neupogen and US-licensed Neupogen, corresponding to two arms of the study, and the third arm corresponds to the biosimilar version Accofil (the test drug $T$ ). The data are actually generated using a threeperiod crossover design. For the purpose of illustrating our methodology, we shall ignore this aspect, and proceed with the assumption of a three-arm parallel design. The sample sizes are $n_{T}=43$ for Accofil, and $n_{R}=43$ for each reference arm. The observed values of the summary statistics (based on the AUC data) are

$$
\bar{x}_{T}=200720.00, \bar{x}_{R_{1}}=192379.97, \bar{x}_{R_{2}}=186404.48, s_{T}=68,244.80, \text { and } s_{R}=60611.94 .
$$

The details given in the European Medicines Agency document [5] indicate that log-normality is reasonable. Since the summary statistics based on the log-transformed data are not available, we proceed assuming normality. The gpq based upper confidence limit for $\theta$ came out to be 15.92 . Thus if we choose a value $\delta=1.20$, the null hypothesis in (1.2) cannot be rejected. In other words, we cannot conclude biosimilarity.

\section{Discussion}

There is increasing interest in the development of biosimilars, and statistical criteria for establishing biosimilarity are still emerging. In the investigation of such criteria, two challenges present themselves: (i) the appropriate criterion/criteria that should be used to establish biosimilarity, and (ii) the development of accurate tests based on the chosen criteria. In this article, we have not advocated any specific statistical criterion; indeed, there is no consensus yet on the statistical criterion that should be used. However, we feel that average biosimilarity is a minimum requirement for a biosimilar 
product. Average biosimilarity has already been investigated in the literature, and can be addressed in the context of two-arm and three-arm parallel designs. The latter set up has recently been taken up by Kang and Chow [7], and our work is also in the same scenario. The contribution in our work is three-fold: we have relaxed the equal variance assumption in their article, we have derived an accurate test using generalized pivotal quantities, and we have suggested an alternative formulation for assessing average biosimilarity. Our test procedures exhibit satisfactory performance in terms of type I error probabilities. Our overall conclusion is that average biosimilarity can be assessed accurately, even when the test and reference formulations exhibit different variabilities.

\section{References}

[1] Y.-W. Chang, Y. Tsong, X. Dong and Z. Zhao (2014). Sample size determination for a three-arm equivalence trial of normally distributed responses. Journal of Biopharmaceutical Statistics, 24, 1190-1202.

[2] S.-C. Chow (2013). Biosimilars: Design and Analysis of Follow-on Biologics, Chapman \& Hall/CRC Press, Boca Raton, Florida.

[3] S.-C. Chow and J. P. Liu (2008). Design and Analysis of Bioavailability and Bioequivalence Studies, Third Edition, Chapman \& Hall/CRC Press, Boca Raton, Florida.

[4] B. Efron and R. Tibshirani (1993). An Introduction to the Bootstrap. Chapman \& Hall/CRC Press, Boca Raton, Florida.

[5] EMA (2014). CHMP Assessment Report: Accofil, Available at http://www.ema.europa.eu.

[6] FDA (2015). Guidance for Industry: Scientific Considerations in Demonstrating Biosimilarity to a Reference Product. The U.S. Food and Drug Administration, Silver Spring, MD.

[7] S. H. Kang and S.-C. Chow (2013). Statistical assessment of biosimilarity based on relative distance between follow-on biologics. Statistics in Medicine, 32, 382-392.

[8] S. H. Kang and Y. Kim (2014). Sample size calculations for the development of biosimilar products. Journal of Biopharmaceutical Statistics, 24, 1215-1224.

[9] K. Krishnamoorthy and T. Mathew (2009). Statistical Tolerance Regions: Theory, Applications and Computation, John Wiley \& Sons, New York.

[10] Pottackal, G. J. (2015). Some Tests, Confidence Limits and Tolerance Limits for Assessing Biosimilarity. Doctoral dissertation submitted to the University of Maryland Baltimore County.

[11] A. Sun, X. Dong and Y. Tsong (2014). Sample size determination for equivalence assessment with multiple endpoints. Journal of Biopharmaceutical Statistics, 24, 1203-1214.

[12] S. Weerahandi (1993). Generalized confidence intervals. Journal of the American Statistical Association, 88, 899-905.

[13] S. Weerahandi (1995). Exact Statistical Methods for Data Analysis. New York: Springer.

[14] S. Weerahandi (2004). Generalized Inference in Repeated Measures: Exact Methods in MANOVA and Mixed Models, John Wiley \& Sons, New York. 